\title{
ON THE SPANS AND WIDTH OF SIMPLE TRIODS
}

\author{
THELMA WEST
}

(Communicated by James West)

\begin{abstract}
In 1964 the concept of the span of a metric space was introduced by A. Lelek. Since that time, some modified versions of the span have been considered. To date, the metric spaces for which the various spans have been explictly calculated have mainly been objects for which the span is zero. In this paper, we calculate and estimate the spans of simple triods.
\end{abstract}

Questions have been raised concerning the relationships between these various versions of spans. For instance, it was conjectured that the surjective span is always at least a half of the span. We find that these spans are equal for some types of simple triods. Another problem concerning simple triods asks if the surjective semispan and the surjective span of them are always equal. This problem is solved positively for some classes of simple triods.

In 1961 the width of a treelike continuum was introduced by C. E. Burgess. The relationship of the width and the span is only vaguely known. We involve the widths of simple triods in our estimations of the span.

In 1964 the concept of the span of a metric space was introduced by A. Lelek. Since that time, some modified versions of the span have been considered (cf. $[2,3]$ ). To date the metric spaces for which the various spans have been explicitly calculated have mainly been objects for which the span is zero. It is still an unsettled problem whether or not continua of span zero are arclike. In this paper we calculate and estimate the spans of simple triods.

Questions have been raised concerning the relationships between these various versions of span. For instance, it was conjectured that the surjective span is always at least a half of the span (see [3, p. 37]). We find that these spans are equal for some types of simple triods (see 2.4). Another problem concerning simple triods asks if the surjective semispan and the surjective span of them are always equal (see [3, p. 38]). This problem is solved positively in $\S 2$ for some classes of simple triods.

In 1961 the width of a treelike continuum was introduced by C. E. Burgess (see $[1$, p. 447]). The relationship of the width and the span is only vaguely

Received by the editors September 25, 1987 and, in revised form, July 28, 1988.

1980 Mathematics Subject Classification (1985 Revision). Primary 54F15; Secondary 54F20. 
known (cf. [4, p. 191 and 6]). We involve the widths of simple triods in our estimations of the span (see 1.8 and 2.4 , for example).

\section{Preliminaries}

All mappings are assumed to be continuous functions. If $f: X \rightarrow Y$ and $g: X \rightarrow Z$ are mappings, the diagonal mapping $f \Delta g: X \rightarrow Y \times Z$ into the product $Y \times Z$ is defined by the formula

$$
(f \Delta g)(x)=(f(x), g(x)), \quad(x \in X),
$$

so that $f \Delta g$ is continuous. The standard projection maps are denoted by $p_{1}$ and $p_{2}$.

Let $X$ be a nonempty metric space. The surjective span $\sigma^{*}(X)$ of $X$ is the least upper bound of real numbers $\alpha$ such that there exist nonempty connected sets $C_{\alpha} \subset X \times X$ with $d(x, y) \geq \alpha$ for $(x, y) \in C_{\alpha}$ and

$$
p_{1}\left(C_{\alpha}\right)=p_{2}\left(C_{\alpha}\right)=X \text {. }
$$

Relaxing condition $\left(\sigma^{*}\right)$ to the conditions

$$
\begin{gathered}
p_{1}\left(C_{\alpha}\right)=p_{2}\left(C_{\alpha}\right), \\
p_{2}\left(C_{\alpha}\right)=X, \\
p_{1}\left(C_{\alpha}\right) \subset p_{2}\left(C_{\alpha}\right),
\end{gathered}
$$

we obtain the definitions of the span $\sigma(X)$, the surjective semispan $\sigma_{0}^{*}(X)$, and the semispan $\sigma_{0}(X)$ of $X$, respectively.

By a continuum we mean a compact, connected metric space.

1.1. If $X$ is a metric space and $\tau=\sigma^{*}, \sigma, \sigma_{0}^{*}, \sigma_{0}$, then $\tau(X)$ is the least upper bound of real numbers $\alpha$ such that there exist nonempty connected sets $C$ and continuous mappings $f, g: C \rightarrow X$ with $d[f(c), g(c)] \geq \alpha$ for $c \in C$ and

$\left(\sigma^{*}\right)^{\prime}$

$(\sigma)^{\prime}$

$\left(\sigma_{0}^{*}\right)^{\prime}$

$\left(\sigma_{0}\right)$

$$
\begin{gathered}
f(C)=g(C)=X, \\
f(C)=g(C), \\
g(C)=X, \\
f(C) \subset g(C),
\end{gathered}
$$

respectively. Moreover, if $X$ is a compact metric space, then $C$ can be assumed to be continua.

1.2. If $X$ is a metric space, then

$$
\begin{aligned}
& 0 \leq \sigma^{*}(X) \leq \sigma(X) \leq \sigma_{0}(X) \leq \operatorname{diam} X, \\
& 0 \leq \sigma^{*}(X) \leq \sigma_{0}^{*}(X) \leq \sigma_{0}(X) \leq \operatorname{diam} X
\end{aligned}
$$

(see [2, p. 207]).

1.3. If $X$ is a metric space and $A \subset X$ is a nonempty subset, then $\sigma(A) \leq \sigma(X)$ and $\sigma_{0}(A) \leq \sigma_{0}(X)$ (see [2, p. 207]). 
1.4. If $X$ is a metric space, $C$ is a continuum, and $f, g: C \rightarrow X$ are mappings satisfying condition $(\tau)^{\prime}$, where $\tau=\sigma^{*}, \sigma, \sigma_{0}^{*}, \sigma_{0}$, then there exists a point $c_{0} \in C$ such that $d\left[f\left(c_{0}\right), g\left(c_{0}\right)\right] \leq \tau(X)$.

1.5. If $X$ is an arc, then $\sigma^{*}(X)=\sigma(X)=\sigma_{0}^{*}(X)=\sigma_{0}(X)=0$ (see [3, p. 36]).

By a simple triod we understand a space $T$ which admits a decomposition $T=A_{0} \cup A_{1} \cup A_{2}$, where $A_{i}$ are arcs having a common endpoint $v$ and $A_{i} \cap A_{i+1}=\{v\}$ for $i=0,1,2$, and the subscripts of $A_{j}$ taken mod 3. The arcs $A_{0}, A_{1}, A_{2}$ are called branches of the simple triod $T$, and the point $v$ is called the branch-point of $T$. The width of $T$ is denoted by $w(T)$.

1.6. If $T$ is a simiple triod with branches $A_{0}, A_{1}, A_{2}$, then

$$
w(T)=\operatorname{Min}\left\{\operatorname{Max}\left\{d\left(x, A_{i+1} \cup A_{i+2}\right): x \in A_{i}\right\}: i=0,1,2\right\},
$$

where the subscripts are taken $\bmod 3($ see $[4$, p. 187]).

1.7. If $T$ is a simple triod, then $w(T) \leq \sigma^{*}(T)$ (see [2, p. 210 , and 5, p. 208]).

For some simple triods, the weak inequality in 1.7 becomes the equality. However, in general, this is not true. It is known that there exists, for each $\varepsilon>0$, a simple triod $T$ on the plane such that $\sigma(T)=1$ and $w\left(T^{\prime}\right)<\varepsilon$ for each simple triod $T^{\prime}$ contained in $T$ (see [4, p. 195]). In such a triod $T$, a subtriod $T^{\prime}$ can be selected with $\sigma^{*}\left(T^{\prime}\right)=\sigma(T)$, whence $\sigma^{*}\left(T^{\prime}\right)=1$. Thus we can have simple triods with surjective span equal 1 and the width as small as one wishes.

We denote the set $\{x \in X: d(a, x) \leq \varepsilon\}$ by $\bar{B}(a, \varepsilon)$.

1.8. If $T$ is a simple triod with a branch-point $v$ and $T^{\prime} \subset T$ is a simple triod such that one branch of $T$ is contained in $\bar{B}\left[v, w\left(T^{\prime}\right)\right]$, then

(i) $\sigma(T)=\sigma_{0}(T)=\sigma\left(T^{\prime}\right)=\sigma_{0}\left(T^{\prime}\right)=w\left(T^{\prime}\right)$,

(ii) $\sigma^{*}\left(T^{\prime}\right)=\sigma_{0}^{*}\left(T^{\prime}\right)=w\left(T^{\prime}\right)$ (see [6]).

\section{Main Results}

2.1 Theorem. Let $T$ be a simple triod with branch point $v$. Assume there exists a simple triod $T^{\prime} \subset T$ such that $\sigma_{0}^{*}(T) \leq w\left(T^{\prime}\right)$. Let $T=L_{0} \cup L_{1} \cup L_{2}$ be the decomposition of $T$ into branches. Assume there exists a branch $L_{k}(k=$ $0,1,2)$ and a homeomorphism $h:[0,1] \rightarrow L_{k}$ of the unit interval $[0,1]$ onto $L_{k}$ such that $h(0)=v$ and the real-valued function $f$ defined by the formula

$$
f(t)=\operatorname{Min}\left\{d[v, h(t)], \sigma_{0}^{*}(T)\right\} \quad(0 \leq t \leq 1)
$$

is nondecreasing (see Figure 1). Then $\sigma_{0}^{*}(T)=\sigma^{*}(T)$.

Proof. Since $\sigma^{*}(T) \leq \sigma_{0}^{*}(T)$, by 1.2 , we will prove the theorem by showing that $\sigma_{0}^{*}(T) \leq \sigma^{*}(T)$. 


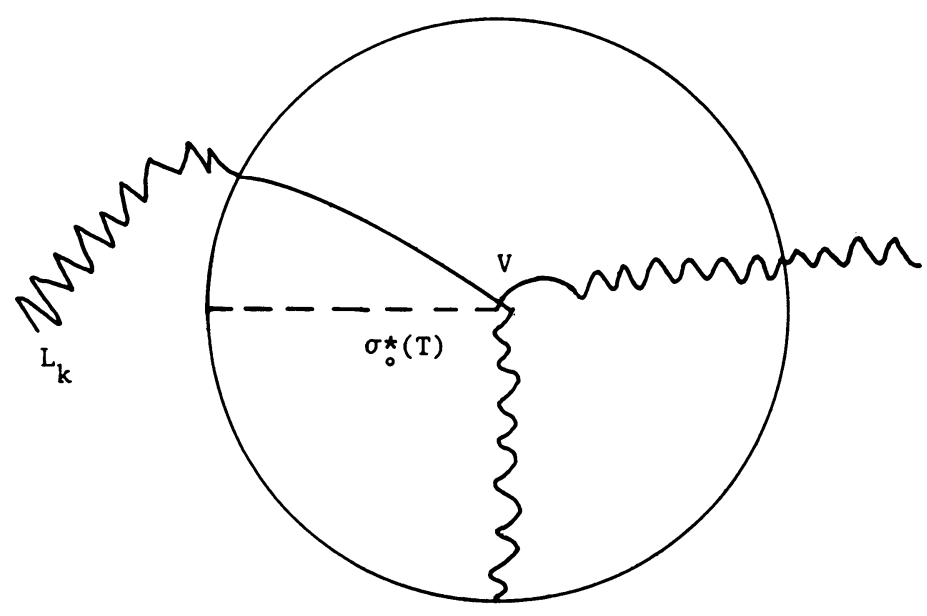

Figure 1.

We observe that $w(T)>0$ by 1.6. Hence, $\sigma_{0}^{*}(T)>0$, since $\sigma_{0}^{*}(T) \geq$ $\sigma^{*}(T)$, by 1.2 , and $\sigma^{*}(T) \geq w(T)$, by 1.7 .

Let $f, g: C \rightarrow T$ be mappings, where $C$ is a continuum,

$$
g(C)=T \quad \text { and } \quad d[f(c), g(c)] \geq \sigma_{0}^{*}(T)-\varepsilon,
$$

for each $c \in C$, where $\varepsilon$ is a real number and $0 \leq \varepsilon<\sigma_{0}^{*}(T)$.

Let $h^{\prime}: T \rightarrow L_{k+1} \cup L_{k+2}$ be the mapping defined by

$$
h^{\prime}(x)= \begin{cases}x & \text { if } x \in L_{k+1} \cup L_{k+2} \\ v & \text { if } x \in L_{k} .\end{cases}
$$

Considering the composites $h^{\prime} f, h^{\prime} g: C \rightarrow T$, we see, by 1.5 , that there is a point $c^{\prime} \in C$ such that $d\left[h^{\prime} f\left(c^{\prime}\right), h^{\prime} g\left(c^{\prime}\right)\right]=0$. It must be the case that $h^{\prime} f\left(c^{\prime}\right)=h^{\prime} g\left(c^{\prime}\right)=v$, otherwise $d\left[f\left(c^{\prime}\right), g\left(c^{\prime}\right)\right]=0$ contrary to (1).

There are three possibilities.

Case 1. $g\left(c^{\prime}\right)=v, \quad f\left(c^{\prime}\right) \in L_{k}-\{v\}$.

Case 2. $f\left(c^{\prime}\right)=v, \quad g\left(c^{\prime}\right) \in L_{k}-\{v\}$.

Case 3. $f\left(c^{\prime}\right) \in L_{k}-\{v\}, \quad g\left(c^{\prime}\right) \in L_{k}-\{v\}$.

In the third case, we observe that the sets $A$ and $B$ defined by the formulas

$$
\begin{aligned}
& A=\left(L_{k}-\{v\}\right) \times\left(L_{k}-\{v\}\right), \\
& B=\left[T \times\left(\left(L_{k+1} \cup L_{k+2}\right)-\{v\}\right)\right] \cup\left[\left(\left(L_{k+1} \cup L_{k+2}\right)-\{v\}\right) \times T\right]
\end{aligned}
$$


are disjoint open subsets of the product $T \times T$. There is a point $c^{\prime \prime} \in C$ such that $g\left(c^{\prime \prime}\right) \in\left(L_{k+1} \cup L_{k+2}\right)-\{v\}$, since $g(C)=T$. Hence, $(f \Delta g)(C) \not \subset(T \times$ $T)-\left(L_{k} \times\{v\} \cup\{v\} \times L_{k}\right)=A \cup B$, since $(f \Delta g)(C) \cap A \neq \varnothing,(f \Delta g)(C) \cap B \neq \varnothing$, and $(f \Delta g)(C)$ is connected. So, there is a point $c^{\prime \prime \prime}$ of $C$ such that either $f\left(c^{\prime \prime \prime}\right)=v$ and $g\left(c^{\prime \prime \prime}\right) \in L_{k}$ or $g\left(c^{\prime \prime \prime}\right)=v$ and $f\left(c^{\prime \prime \prime}\right) \in L_{k}$. Also, it is not the case that $g\left(c^{\prime \prime \prime}\right)=f\left(c^{\prime \prime \prime}\right)=v$, since, by (1), $d[f(c), g(c)]>0$, for each $c \in C$.

In all three cases, we have established the existence of a point $c \in C$ and a point $l \in L_{k}-\{v\}$ such that either $f(c)=v$ and $g(c)=l$ or $g(c)=v$ and $f(c)=l$.

Let $t^{\prime}=\operatorname{Min}\left\{t \in I: d\left[h(t), L_{k+1}^{\prime} \cup L_{k+2}^{\prime}\right] \geq w\left(T^{\prime}\right)\right\}$, where $T^{\prime}=L_{0}^{\prime} \cup L_{1}^{\prime} \cup L_{2}^{\prime}$ and $L_{i}^{\prime} \subset L_{i}$ for $i=0,1,2$. By 1.6, we know that $t^{\prime}$ does exist. Let $a_{k}=$ $h\left(t^{\prime}\right)$. Let $t^{\prime \prime}=h^{-1}(l)$.

We must consider two cases as follows:

Case 1. $t^{\prime \prime} \leq t^{\prime}$. Let $A=h\left(\left[t^{\prime \prime}, t^{\prime}\right]\right)$. For $t \in\left[t^{\prime \prime}, t^{\prime}\right], d[v, h(t)] \geq \sigma_{0}^{*}(T)-\varepsilon$, since $d\left[v, h\left(t^{\prime \prime}\right)\right] \geq \sigma_{0}^{*}(T)-\varepsilon$ and $f$ is a nondecreasing function.

Case 2. $t^{\prime} \leq t^{\prime \prime}$. Let $A=h\left(\left[t^{\prime}, t^{\prime \prime}\right]\right)$. For $t \in\left[t^{\prime}, t^{\prime \prime}\right], d[v, h(t)] \geq \sigma_{0}^{*}(T)$, since $d\left[v, h\left(t^{\prime}\right)\right] \geq w\left(T^{\prime}\right) \geq \sigma_{0}^{*}(T)$ and $f$ is a nondecreasing function.

In both cases we have established that

$$
d(v, A) \geq \sigma_{0}^{*}(T)-\varepsilon .
$$

Pick points $a_{k+1} \in L_{k+1}^{\prime}$ and $a_{k+2} \in L_{k+2}^{\prime}$ such that

$$
\begin{aligned}
& d\left(a_{k+1}, L_{k}^{\prime} \cup L_{k+2}^{\prime}\right) \geq w\left(T^{\prime}\right), \\
& d\left(a_{k+2}, L_{k}^{\prime} \cup L_{k+1}^{\prime}\right) \geq w\left(T^{\prime}\right) .
\end{aligned}
$$

We are able to pick such points by 1.6. Let $A_{k+1}$ be the subarc of $L_{k+1}$ with endpoints $v$ and $a_{k+1}$. Let $A_{k+2}$ be the subarc of $L_{k+2}$ with endpoints $v$ and $a_{k+2}$.

We define a set $D$ in $T \times T$ by the following formula:

$$
\begin{aligned}
& D=[\{v\} \times A] \cup\left[A_{k+1} \times\left\{a_{k}\right\}\right] \cup\left[\left\{a_{k+1}\right\} \times\left(A_{k} \cup A_{k+2}\right)\right] \\
& \cup {\left[\left(A_{k+1} \cup A_{k}\right) \times\left\{a_{k+2}\right\}\right] \cup\left[\left\{a_{k}\right\} \times A_{k+2}\right] \cup[A \times\{v\}] . }
\end{aligned}
$$

Notice that $D$ is connected, since each of the six sets in the union is an arc or a point, and each of the first five sets in the union intersects the succeeding set.

Since $\sigma_{0}^{*}(T) \leq w\left(T^{\prime}\right)$, and by (2) and (3), we see that if $(x, y) \in D$, then

$$
d(x, y) \geq \sigma_{0}^{*}(T)-\varepsilon .
$$

We define a set $C_{\alpha}$ in $T \times T$ by

$$
C_{\alpha}=(f \Delta g)(C) \cup D \cup(g \Delta f)(C) .
$$


This set is connected since $(f \Delta g)(C), D$, and $(g \Delta f)(C)$ are each connected, and $(f \Delta g)(C) \cap D \neq \varnothing$ and $(g \Delta f)(C) \cap D \neq \varnothing$. Also, $p_{1}\left(C_{\alpha}\right)=$ $p_{2}\left(C_{\alpha}\right)=T$, since $g(C)=T$. If $(x, y) \in C_{\alpha}$, then by (1) and (4) we see that $d(x, y) \geq \sigma_{0}^{*}(T)-\varepsilon$. From the definition of the surjective span, we see that $\sigma^{*}(T) \geq \sigma_{0}^{*}(T)-\varepsilon$ and since $\varepsilon$ was arbitrarily small, we have $\sigma^{*}(T) \geq \sigma_{0}^{*}(T)$.

2.2 Corollary. Let $T$ be a simple triod with a branch-point $v$ and let $T^{\prime} \subset T$ be a simple triod such that one branch of $T$ is contained in $\bar{B}\left[v, w\left(T^{\prime}\right)\right]$. Let $T=L_{0} \cup L_{1} \cup L_{2}$ be the decomposition of $T$ into branches. Assume there exists a branch $L_{k}(k=0,1,2)$ and a homeomorphism $h:[0,1] \rightarrow L_{k}$ of the unit interval $[0,1]$ onto $L_{k}$ such that $h(0)=v$ and the real-valued function $f$ defined by the formula

$$
f(t)=\operatorname{Min}\left\{d[v, h(t)], \sigma_{0}^{*}(T)\right\} \quad(0 \leq t \leq 1)
$$

is nondecreasing (see Figure 1). Then $\sigma_{0}^{*}(T)=\sigma^{*}(T)$.

Proof. By 1.8 we have that $\sigma_{0}(T)=w\left(T^{\prime}\right)$. Since $\sigma_{0}^{*}(T) \leq \sigma_{0}(T)$, by 1.2 , we have $\sigma_{0}^{*}(T) \leq w\left(T^{\prime}\right)$. Consequently, the corollary follows.

The following lemma and proof are due to A. Lelek.

2.3 Lemma. Let $p_{0}, p_{1}, p_{2}$ be infinite rays in the plane $R^{2}$, i.e., homeomorphic copies of the half-line $[0, \infty)$, such that the origin $O$ is an endpoint of each of them and the only point belonging to any two of them, and their union $P_{0} \cup P_{1} \cup P_{2}$ cuts the plane $R^{2}$ into three regions $Q_{0}, Q_{1}, Q_{2}$ (see Figure 2). Let $C \subset R^{2} \backslash\{O\}$ be a connected set. Let $C_{0}, C_{1}, C_{2}$ be connected subsets of a space $X$ with points $a_{i+1}, a_{i+2} \in C_{i}(i=0,1,2)$, where the subscripts are taken mod 3 . Then the subset $Y$ of the product $X \times R^{2}$ defined by the formula

$$
Y=\bigcup_{i=0}^{2}\left\{\left[C_{i} \times\left(C \cap P_{i}\right)\right] \cup\left[\left\{a_{i}\right\} \times\left(C \cap Q_{i}\right)\right]\right\}
$$

is connected.

Proof. Suppose $Y=A \cup B$, where $A \neq \varnothing \neq B$. Then

$$
\begin{aligned}
C=C \cap R^{2} & =C \cap \bigcup_{i=0}^{2}\left(P_{i} \cup Q_{i}\right)=\bigcup_{i=0}^{2}\left[\left(C \cap P_{i}\right) \cup\left(C \cap Q_{i}\right)\right] \\
& =\bigcup_{i=0}^{2}\left\{p_{2}\left[C_{i} \times\left(C \cap P_{i}\right)\right] \cup p_{2}\left[\left\{a_{i}\right\} \times\left(C \cap Q_{i}\right)\right]\right\} \\
& =p_{2}(Y)=p_{2}(A \cup B)=p_{2}(A) \cup p_{2}(B)
\end{aligned}
$$

and, since $C$ is connected, we have $p_{2}(A) \cap \mathrm{cl} p_{2}(B) \neq \varnothing$ or $p_{2}(B) \cap \mathrm{cl} p_{2}(A) \neq$ $\varnothing$. Without loss of generality, we can assume that $p_{2}(A) \cap \mathrm{Cl} p_{2}(B) \neq \varnothing$. Hence there exists a point $q \in C$ such that $q \in p_{2}(A)$ and $q \in \operatorname{cl} p_{2}(B)$.

Case 1. $q \in Q_{i}(i=0,1,2)$. The set $Q_{i}$ is open in $R^{2}$, whence $q$ belongs to the closure of $p_{2}(B) \cap Q_{i}$. But

$$
p_{2}^{-1}\left(Q_{i}\right) \cap Y=\left(X \times Q_{i}\right) \cap Y=\left\{a_{i}\right\} \times\left(C \cap Q_{i}\right)
$$




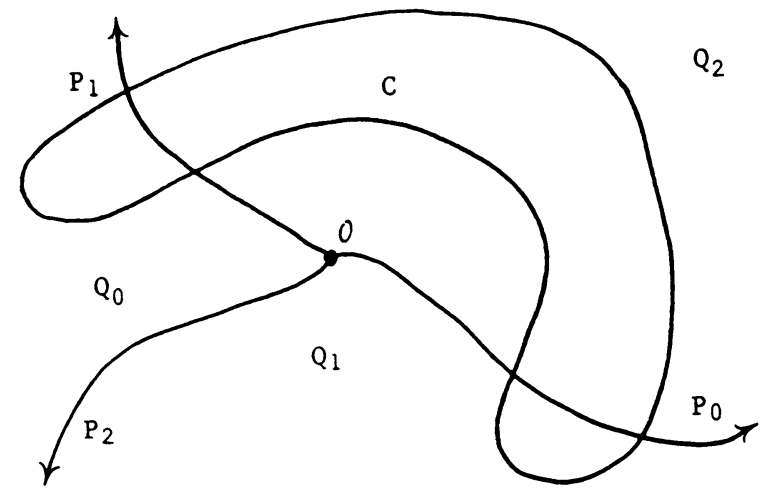

FIGURE 2.

which implies $p_{2}^{-1}(q) \cap Y=\left\{\left(a_{i}, q\right)\right\}$ and $\left(a_{i}, q\right) \in A$, since $q \in p_{2}(A)$. Also, by (5), we have $p_{2}^{-1}\left(q^{\prime}\right) \cap Y=\left\{\left(a_{i}, q^{\prime}\right)\right\}$ for any other point $q^{\prime}$ of $C \cap Q_{i}$. Thus, in particular, $\left(a_{i}, q^{\prime}\right) \in B$ for $q^{\prime} \in p_{2}(B) \cap Q_{i}$; that is, $\left\{a_{i}\right\} \times\left[p_{2}(B) \cap Q_{i}\right]$ is a subset of $B$. However, the point $\left(a_{i}, q\right)$ belongs to the closure of this subset in $X \times R^{2}$, whence $\left(a_{i}, q\right) \in \operatorname{cl} B$. Therefore, $\left(a_{i}, q\right)$ belongs to $A \cap \operatorname{cl} B$.

Case 2. $q \notin Q_{0} \cup Q_{1} \cup Q_{2}$. Then $q \in P_{j}(j=0,1,2)$, and since $q \in C \subset$ $R^{2} \backslash\{O\}$, the point $q$ is not the endpoint $O$ of the ray $P_{j}$. Consequently, the set $P_{j} \cup Q_{j+1} \cup Q_{j+2}$ is a neighborhood of $q$ in $R^{2}$. As a result, $q \in \operatorname{cl} p_{2}(B)$ implies that $q$ belongs to the closure of the common part of $p_{2}(B)$ with this neighborhood, so that

$$
q \in \operatorname{cl}\left[p_{2}(B) \cap P_{j}\right] \cup \operatorname{cl}\left[p_{2}(B) \cap Q_{j+1}\right] \cup \operatorname{cl}\left[p_{2}(B) \cap Q_{j+2}\right] .
$$

Case 2(a). $q \in \operatorname{cl}\left[p_{2}(B) \cap P_{j}\right]$ and $p_{2}^{-1}\left(q^{\prime}\right)$ does not meet both sets $A$ and $B$ for any $q^{\prime} \in C \cap P_{j}$. Observe, again, that $q^{\prime} \in C \cap P_{j}$ implies $q^{\prime} \neq 0$. By the definition of $Y$, we have

$$
p_{2}^{-1}\left(q^{\prime}\right) \cap Y=\left(X \times\left\{q^{\prime}\right\}\right) \cap Y=C_{j} \times\left\{q^{\prime}\right\} \quad \text { for } q^{\prime} \in C \cap P_{j},
$$

and $a_{j+1} \in C_{j}$ according to the assumption of the lemma. Since $q \in p_{2}(A)$, the set $p_{2}^{-1}(q)$ meets $A$, so it does not meet $B$ as $q \in C \cap P_{j}$. Also, $\left(a_{j+1}, q\right) \in$ $p_{2}^{-1}(q) \cap Y$, by $(6)$; hence $\left(a_{j+1}, q\right) \in A$. On the other hand, the set $p_{2}^{-1}\left(q^{\prime}\right)$ meets $B$ for $q^{\prime} \in p_{2}(B) \cap P_{j}$, so it does not meet $A$ as $p_{2}(B) \subset C$. But 
$\left(a_{j+1}, q^{\prime}\right) \in p_{2}^{-1}\left(q^{\prime}\right) \cap Y$ for each such point $q^{\prime}$, by $(6)$; hence $\left(a_{j+1}, q^{\prime}\right) \in B$. We conclude that $\left\{a_{j+1}\right\} \times\left[p_{2}(B) \cap P_{j}\right]$ is a subset of $B$. However, the point $\left(a_{j+1}, q\right)$ belongs to the closure of this subset in $X \times R^{2}$, whence $\left(a_{j+1}, q\right) \in$ $\operatorname{cl} B$. Therefore, $\left(a_{j+1}, q\right)$ belongs to $A \cap \operatorname{cl} B$.

Case 2(b). $q \in \operatorname{cl}\left[p_{2}(B) \cap Q_{j+1}\right]$ and $p_{2}^{-1}(q)$ does not meet both sets $A$ and $B$. As in Case 2(a), we have $\left(a_{j+1}, q\right) \in A$. As in Case 1, we have

$$
\left\{a_{j+1}\right\} \times\left[p_{2}(B) \cap Q_{j+1}\right] \subset B,
$$

and now $\left(a_{j+1}, q\right)$ belongs to the closure of this subset, whence it belongs to $A \cap \mathrm{cl} B$ again.

Case 2(c). $q \in \operatorname{cl}\left[p_{2}(B) \cap Q_{j+2}\right]$ and $p_{2}^{-1}(q)$ does not meet both sets $A$ and $B$. Since $a_{j+2} \in C_{j}$ according to the assumption of the lemma, we can replace $a_{j+1}$ by $a_{j+2}$ in Case 2(a) to infer that $\left(a_{j+2}, q\right) \in A$. Similarly, as in Case 1, we have

$$
\left\{a_{j+2}\right\} \times\left[p_{2}(B) \cap Q_{j+2}\right] \subset B,
$$

and thus, in this case, the point $\left(a_{j+2}, q\right)$ belongs to $\operatorname{cl} B$, hence also to $A \cap \mathrm{ncl} B$. Case 2(d). $p_{2}^{-1}\left(q^{\prime}\right)$ meets both sets $A$ and $B$ for at least one point $q^{\prime} \in C \cap P_{j}$. Then, by (6) we obtain

$$
C_{j} \times\left\{q^{\prime}\right\}=p_{2}^{-1}\left(q^{\prime}\right) \cap Y=p_{2}^{-1}\left(q^{\prime}\right) \cap(A \cup B)=\left[p_{2}^{-1}\left(q^{\prime}\right) \cap A\right] \cup\left[p_{2}^{-1}\left(q^{\prime}\right) \cap B\right],
$$

where both sets in the brackets are nonempty. But $C_{j} \times\left\{q^{\prime}\right\}$ is a connected set, which implies that

$$
\varnothing \neq\left[p_{2}^{-1}\left(q^{\prime}\right) \cap A\right] \cap \mathrm{cl}\left[p_{2}^{-1}\left(q^{\prime}\right) \cap B\right] \subset A \cap \mathrm{cl} B
$$

or

$$
\varnothing \neq\left[p_{2}^{-1}\left(q^{\prime}\right) \cap B\right] \cap \mathrm{cl}\left[p_{2}^{-1}\left(q^{\prime}\right) \cap A\right] \subset B \cap \operatorname{cl} A .
$$

In all cases, we have shown that $A \cap \operatorname{cl} B \neq \varnothing$ or $B \cap \operatorname{cl} A \neq \varnothing$. Consequently, the set $Y$ is connected, and 2.3 is proved.

2.4 Theorem. Let $T \subset R^{2}$ be a simple triod in the plane $R^{2}$, let $v$ be the branch-point of $T$, and let $T^{\prime} \subset T$ be a simple triod such that one branch of $T$ is contained in $\bar{B}\left[v, w\left(T^{\prime}\right)\right]$. Let $T^{\prime}=L_{0}^{\prime} \cup L_{1}^{\prime} \cup L_{2}^{\prime}$ be the decomposition of $T^{\prime}$ into branches. Let $a_{i} \in L_{i}^{\prime}$ be points such that

$$
d\left(a_{i}, L_{i+1}^{\prime} \cup L_{i+2}^{\prime}\right) \geq w\left(T^{\prime}\right) \quad(i=0,1,2),
$$

where the subscripts are taken $\bmod 3$ (the existence of such points follows from 1.6). Assume there exist pairwise disjoint infinite rays $M_{i}$ in the plane $R^{2}$ such that $a_{i}$ is an endpoint of $M_{i}$ and

$$
B\left[q, w\left(T^{\prime}\right)\right] \cap\left(L_{i+1}^{\prime} \cup L_{i+2}^{\prime}\right)=\varnothing \quad\left(q \in M_{i}, i=0,1,2\right) .
$$


Then $\sigma(T)=\sigma_{0}(T)=\sigma^{*}(T)=\sigma_{0}^{*}(T)=w\left(T^{\prime}\right)$.

Proof. By 1.8 we have $\sigma(T)=\sigma_{0}(T)=w\left(T^{\prime}\right)$, and, in view of 1.2 , it is enough to show that $\sigma^{*}(T) \geq w\left(T^{\prime}\right)$.

Let $H_{i}$ be the subarc of $L_{i}^{\prime}$ with endpoints $v$ and $a_{i}$ for $i=0,1,2$. We can assume that $M_{i} \cap H_{i}=\left\{a_{i}\right\}$ for $i=0,1,2$. There is a homeomorphism $m_{i}$ from $[0, \infty+)$ onto $M_{i}$, for each $i$. Let $t_{i}=\max \left\{t: t \in[0, \infty+), m_{i}(t) \in H_{i}\right\}$ for $i=0,1,2$. We could have chosen $a_{i}$ to be $m_{i}\left(t_{i}\right)$, for $i=0,1,2$. This is the case since $d\left[m_{i}\left(t_{i}\right), L_{i+1}^{\prime} \cup L_{i+2}^{\prime}\right] \geq w\left(T^{\prime}\right)$ because $m_{i}\left(t_{i}\right) \in M_{i}$ for each $i$. Also, $m_{i}\left(\left[t_{i}, \infty+\right)\right)$, where $i=0,1,2$, are pairwise disjoint infinite rays.

For each $i, H_{i} \cup M_{i}$ is an infinite ray with endpoint $v$. This is the case, since $H_{i}$ is an arc with endpoints $v$ and $a_{i}, M_{i}$ is an infinite ray with endpoint $a_{i}$, and $H_{i} \cap M_{i}=\left\{a_{i}\right\}$ for $i=0,1,2$.

For each $i, M_{i+2} \cup H_{i+2} \cup H_{i+1} \cup M_{i+1}$ is a line in the plane. This is true since $\left(M_{i+2} \cup H_{i+2}\right) \cap\left(H_{i+1} \cup M_{i+1}\right)=\{v\}$ and $v$ is the common endpoint of the infinite rays $M_{i+2} \cup H_{i+2}$ and $H_{i+1} \cup M_{i+1}$. Hence, $R^{2}-\left(M_{i+2} \cup H_{i+2} \cup\right.$ $\left.H_{i+1} \cup M_{i+1}\right)$ consists of two components. We label the component which does not intersect $\left(H_{i} \cup M_{i}\right), Q_{i}$.

Let $H_{0}^{\prime}, H_{1}^{\prime}, H_{2}^{\prime}$ be the arcs or points such that $H_{i} \cap H_{i}^{\prime}=\left\{a_{i}\right\}$ for $i=0,1,2$ and $T=\bigcup_{i=0}^{2}\left(H_{i} \cup H_{i}^{\prime}\right)$.

We define subsets of $T \times T$ by the following formulas:

$$
\begin{gathered}
A_{i}=\left\{a_{i}\right\} \times\left(H_{i+1} \cup H_{i+2}\right), \\
A_{i j}=\left(H_{i}^{\prime} \cap Q_{j}\right) \times\left\{a_{j}\right\}, \\
B_{i j}=\left(H_{i}^{\prime} \cap M_{j}\right) \times\left(H_{j+1} \cup H_{j+2}\right), \\
A_{i}^{\prime}=\left(H_{i+1} \cup H_{i+2}\right) \times\left\{a_{i}\right\}, \\
A_{i j}^{\prime}=\left\{a_{j}\right\} \times\left(H_{i}^{\prime} \cap Q_{j}\right), \\
B_{i j}^{\prime}=\left(H_{j+1} \cup H_{j+2}\right) \times\left(H_{i}^{\prime} \cap M_{j}\right), \\
C_{\alpha}=\bigcup_{i=0}^{2}\left\{A_{i} \cup A_{i}^{\prime} \cup\left[\bigcup_{j=0}^{2}\left(A_{i j} \cup A^{\prime} i j\right)\right] \cup\left[\bigcup_{j=0}^{2}\left(B_{i j} \cup B_{i j}^{\prime}\right)\right]\right\} .
\end{gathered}
$$

We see that $H_{i+1} \cup H_{i+2} \subset p_{2}\left(C_{\alpha}\right)$, for each $i$, from (7). Hence, $H_{0} \cup H_{1} \cup$ $H_{2} \subset p_{2}\left(C_{\alpha}\right)$. Similarly, from (10), we see that $H_{0} \cup H_{1} \cup H_{2} \subset p_{1}\left(C_{\alpha}\right)$.

We observe that $R^{2}=\bigcup_{j=0}^{2}\left(Q_{j} \cup M_{j} \cup H_{j}\right)$. Hence, if $y \in H_{i}^{\prime}$, for any $i$, then there is a $j \in\{0,1,2\}$ such that $y \in Q_{j} \cup M_{j}$. Hence, $H_{i}^{\prime} \subset p_{1}\left(C_{\alpha}\right)$, for each $i$, by (8) and (9). Consequently, $H_{0}^{\prime} \cup H_{1}^{\prime} \cup H_{2}^{\prime} \subset p_{1}\left(C_{\alpha}\right)$. Similarly, we see, by (11) and (12) that $H_{0}^{\prime} \cup H_{1}^{\prime} \cup H_{2}^{\prime} \subset p_{2}\left(C_{\alpha}\right)$. Whence, $T=p_{1}\left(C_{\alpha}\right)=p_{2}\left(C_{\alpha}\right)$. 
To show that $C_{\alpha}$ is connected, we employ Lemma 2.3. We define $Y_{i}$ for $i=0,1,2$ by

$$
Y_{i}=\bigcup_{j=0}^{2}\left\{\left[\left(H_{j+1} \cup H_{j+2}\right) \times\left(H_{i}^{\prime} \cap\left(H_{j} \cup M_{i}\right)\right)\right] \cup\left[\left\{a_{j}\right\} \times\left(H_{i}^{\prime} \cap Q_{j}\right)\right]\right\} .
$$

To see that we can apply 2.3 to conclude that $Y_{i}$ is a connected subset of $T \times R^{2}$, we make the following observations. The set $H_{j+1} \cup H_{j+2}$ is an arc, hence it is a connected subset of $T$. Also, $a_{j+1}, a_{j+2} \in H_{j+1} \cup H_{j+2}$. So, we let $C_{j}=H_{j+1} \cup H_{j+2}$ for $j=0,1,2$. We have already determined that $H_{j} \cup M_{j}$ is an infinite ray, and that $\left(H_{j} \cup M_{j}\right) \cap\left(H_{j+1} \cup M_{j+1}\right)=\{v\}$ for $j=0,1,2$. So, we let $P_{j}=H_{j} \cup M_{j}$ for $j=0,1,2$. The set $H_{i}^{\prime}$ is an arc and $v \notin H_{i}^{\prime}$. So we let $C=H_{i}^{\prime}$.

To see that $C_{\alpha}$ is connected, we need the following observations:

$$
\begin{gathered}
Y_{i}=\bigcup_{j=0}^{2}\left\{\left[H_{j+1} \cup H_{j+2}\right) \times\left(H_{i}^{\prime} \cap H_{j}\right)\right] \cup\left[\left(H_{j+1} \cup H_{j+2}\right) \times\left(H_{i}^{\prime} \cap M_{j}\right)\right] \\
\left.\cup\left[\left\{a_{j}\right\} \times\left(H_{i}^{\prime} \cap Q_{j}\right)\right]\right\}=A_{i}^{\prime} \cup\left[\bigcup_{j=0}^{2}\left(B_{i j}^{\prime} \cup A_{i j}^{\prime}\right)\right] .
\end{gathered}
$$

Similarly, the set $Y_{i}^{T}$ defined by

$$
Y_{i}^{T}=\bigcup_{j=0}^{2}\left\{\left[\left(H_{i}^{\prime} \cap\left(H_{j} \cup M_{j}\right)\right) \times\left(H_{j+1} \cup H_{j+2}\right)\right] \cup\left[\left(H_{i} \cap Q_{j}\right) \times\left\{a_{j}\right\}\right]\right\}
$$

is connected for $i=0,1,2$. Also,

$$
\begin{aligned}
Y_{i}^{T}= & \bigcup_{j=0}^{2}\left\{\left[\left(H_{i}^{\prime} \cap H_{j}\right) \times\left(H_{j+1} \cup H_{j+2}\right)\right] \cup\left[\left(H_{i}^{\prime} \cap M_{j}\right) \times\left(H_{j+1} \cup H_{j+2}\right)\right]\right. \\
& \left.\cup\left[\left(H_{i}^{\prime} \cap Q_{j}\right) \times\left\{a_{j}\right\}\right]\right\}=A_{i} \cup\left[\bigcup_{j=0}^{2}\left(B_{i j} \cup A_{i j}\right)\right]
\end{aligned}
$$

So, $C_{\alpha}$ can be written as

$$
C_{\alpha}=Y_{0} \cup Y_{1}^{T} \cup Y_{2} \cup Y_{0}^{T} \cup Y_{1} \cup Y_{2}^{T} .
$$

Hence, $C_{\alpha}$ is the union of these six connected sets. Also, $\left(a_{1}, a_{0}\right) \in Y_{0} \cap Y_{1}^{T}$, $\left(a_{1}, a_{2}\right) \in Y_{1}^{T} \cap Y_{2},\left(a_{0}, a_{2}\right) \in Y_{2} \cap Y_{0}^{T},\left(a_{0}, a_{1}\right) \in Y_{0}^{T} \cap Y_{1}$, and $\left(a_{2}, a_{1}\right) \in$ $Y_{1} \cap Y_{2}^{T}$. Whence, $C_{\alpha}$ is connected.

Next, we will show that if $(x, y) \in C_{\alpha}$, then $d(x, y) \geq w\left(T^{\prime}\right)$. We distinguish six cases as follows.

Case 1. $x=a_{i}$ and $y \in H_{i+1} \cup H_{i+2}$. Clearly, $d(x, y) \geq w\left(T^{\prime}\right)$, since $d\left(a_{i}, L_{i+1}^{\prime} \cup L_{i+2}^{\prime}\right) \geq w\left(T^{\prime}\right)$ and $H_{i+1} \cup H_{i+2} \subset L_{i+1}^{\prime} \cup L_{i+2}^{\prime}$. 
Case 2. $x \in Q_{j}$ and $y=a_{j}$. The straight line segment in the plane with endpoints $a_{j}$ and $x$ must cross $M_{j+1} \cup H_{j+1} \cup H_{j+2} \cup M_{j+2}$ at some point, say $z$. If $z \in M_{j+1} \cup M_{j+2}$, then $d\left(z, a_{j}\right) \geq w\left(T^{\prime}\right)$. Since $B\left[z, w\left(T^{\prime}\right)\right] \cap\left(L_{j}^{\prime} \cup\right.$ $\left.L_{j+2}^{\prime}\right)=\varnothing$ for $z \in M_{j+1}$ and $B\left[z, w\left(T^{\prime}\right)\right] \cap\left(L_{j}^{\prime} \cup L_{j+1}^{\prime}\right)=\varnothing$ for $z \in M_{j+2}$. Hence, $d(x, y) \geq w\left(T^{\prime}\right)$, since $d(x, y) \geq d\left(z, a_{j}\right)$. If $z \in H_{j+1} \cup H_{j+2}$, then $d\left(z, a_{j}\right) \geq w\left(T^{\prime}\right)$, since $d\left(a_{j}, L_{j+1}^{\prime} \cup L_{j+2}^{\prime}\right) \geq w\left(T^{\prime}\right)$ and $H_{j+1} \cup H_{j+2} \subset$ $L_{j+1}^{\prime} \cup L_{j+2}^{\prime}$. Hence, $d(x, y) \geq w\left(T^{\prime}\right)$, since $d(x, y) \geq d\left(z, a_{j}\right)$.

Case 3. $x \in M_{j}$ and $y \in H_{j+1} \cup H_{j+2}$. Since $B\left[x, w\left(T^{\prime}\right)\right] \cap\left(L_{i+1}^{\prime} \cup L_{i+2}^{\prime}\right)=\varnothing$ and $H_{j+1} \cup H_{j+2} \subset L_{i+1}^{\prime} \cup L_{i+2}^{\prime}$, we have $d(x, y) \geq w\left(T^{\prime}\right)$.

Case 4. $x \in H_{i+1} \cup H_{i+2}$ and $y=a_{j}$. From Case 1, it follows that $d(x, y) \geq$ $w\left(T^{\prime}\right)$.

Case 5. $x=a_{j}$ and $y \in Q_{j}$. From Case 2, it follows that $d(x, y) \geq w\left(T^{\prime}\right)$.

Case 6. $x \in H_{j+1} \cup H_{j+2}$ and $y \in M_{j}$. From Case 3, it follows that $d(x, y) \geq$ $w\left(T^{\prime}\right)$.

In all six cases we have shown that $d(x, y) \geq w\left(T^{\prime}\right)$. Hence $\sigma^{*}(T) \geq w\left(T^{\prime}\right)$.

\section{REFERENCES}

1. C. E. Burgess, Collections and sequences of continua in the plane II, Pacific J. Math. 11 (1961), 447-454.

2. A. Lelek, An example of a simple triod with surjective span smaller than span, Pacific J. Math. 64 (1976), 207-215.

3. __ On the surjective span and semispan of connected metric spaces, Colloq. Math. 37 (1977), 35-45.

4. _ The span and the width of continua, Fund. Math. 98 (1978), 181-199.

5. A. Lelek and L. Mohler, Real-valued continuous functions and the span of continua, Colloq. Math. 32 (1975), 207-209.

6. T. West, Spans of simple triods, Proc. Amer. Math. Soc. 102 (2) (1988), 407-415.

Department of Mathematics, University of Southwestern Louisiana, Lafayette, LOUISIANA 70504 